\title{
A double-edged sword
}

Turning signalling pathways off can be just as important as switching them on. Many signalling pathways contain a negative feedback loop, which controls the duration of the signal and prevents overstimulation. One way to cut short a signal is to decrease surface levels of receptors after they have been engaged by their ligand. After ligand-activation, several receptor tyrosine kinases are ubiquitinated, then internalized by clathrin-dependent endocytosis and finally degraded. Now two reports clarify how the epidermal growth-factor receptor (EGFR) and the hepatocyte growth-factor receptor (HGFR) are endocytosed from the cell surface (Nature 416, 183-187 and Nature 416, 187-190; 2002). Each group addressed the question from a different angle - Ivan Dikic's group looked for new partners for Cbl, whereas Silvia Giordano's group looked for new partners for endophilins — but in both cases they found the same protein: CIN85. So how are these three proteins connected? $\mathrm{Cbl}$ is an effector of several growth-factor receptors, and has also been shown to ubiquitinate active receptors through its RING domain, thus targeting them for degradation. Endophilins are lipid enzymes that influence membrane curvature and regulate the formation of clathrincoated pits. Both teams detected a tripartite complex of $\mathrm{Cbl}$, CIN85 and endophilin in mammalian cells. They also showed that $\mathrm{Cbl}$ is recruited to the EGFR and phosphorylated in an EGFinduced manner, which in turn increases binding of CIN85 to the distal carboxyl-terminus of Cbl. CIN85 also binds to endophilins, which leads to their recruitment to activated receptors (see figure). In addition, Giordano and colleagues showed that in cells expressing a constitutively active HGFR, endophilins are constitutively associated with the HGFR via a Cbl/CIN85 complex. To show that these interactions are required to decrease the number of EGFRs at the cell surface, Dikic and colleagues generated dominant-negative CIN85 constructs, which fail to interact with $\mathrm{Cbl}$ or endophilin. Overexpressing these mutants

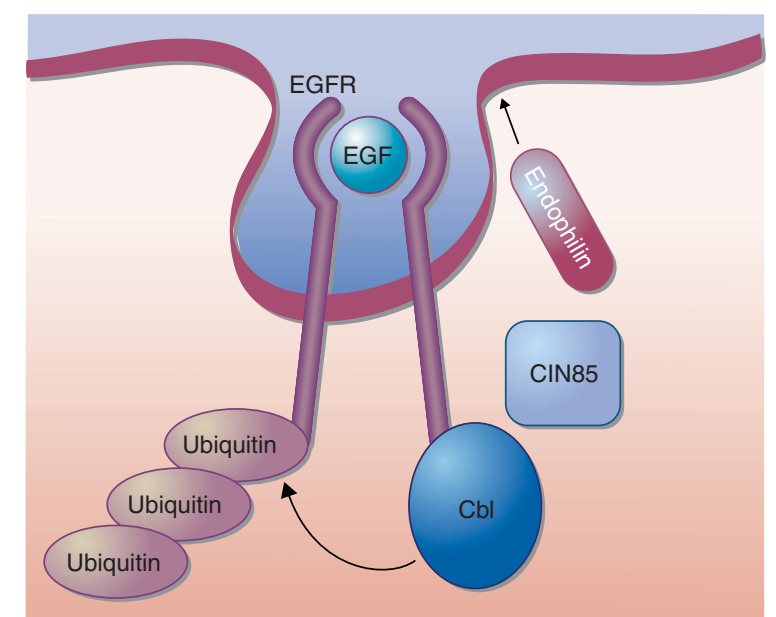

blocks internalization of the EGFR, delays its degradation, and enhances EGF-dependent transcription. Conversely, when Giordano overexpressed the same construct, they found prolonged HGFR phosphorylation, and increased HGFR signalling. So, Cbl, because it can interact with different partners and connect growth factor receptors to both downstream signalling molecules and to regulators of endocytosis, is both an effector and a negative regulator of growth-factor receptors.

VALERIE FERRIER

\section{News and Views contributions}

The News and Views section provides a forum in which new advances in the field of cell biology, as reported in published papers, can be communicated to a wide audience.

Most News and Views pieces are linked to Articles that appear in Nature Cell Biology, but some may focus on papers of exceptional significance that are published elsewhere. Unsolicited contributions will not normally be considered, although prospective authors are welcome to make proposals to the Editor before the paper is published.
As a general guideline, News and Views pieces should be about 1,300 words, with one or two display items (figures, boxes and tables). They should make clear the advance (the 'news') and communicate a sense of excitement, yet provide a critical evaluation of the work in context of the rest of the field. We encourage personal 'views', criticisms and predictions, but authors should not refer to their own work, except in passing. Detailed guidelines are available on request from cellbio@nature.com and on Nature Cell Biology's Web site (http://cellbio.nature.com). 